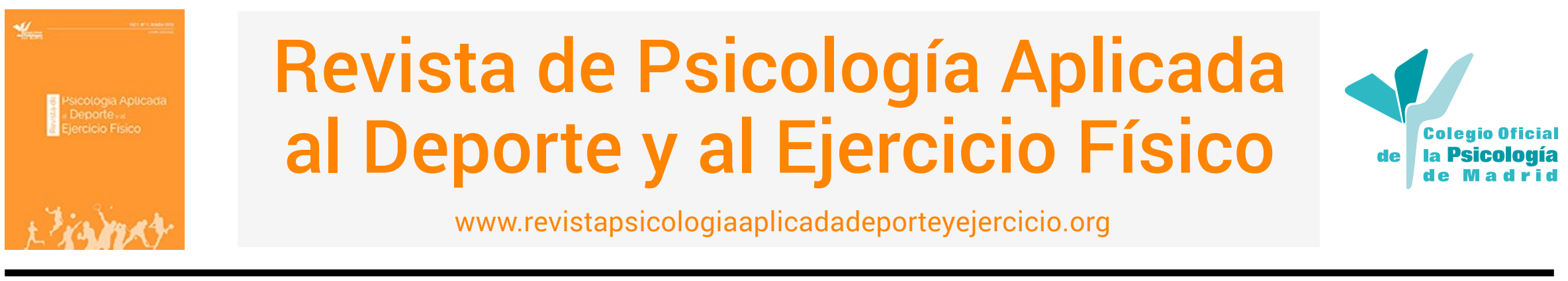

\title{
Intervención psicológica a distancia con un futbolista profesional: estudio de caso
}

\author{
David Peris-Delcampo \\ Universidad de Valencia, España
}

RESUMEN: El objetivo del presente trabajo es estudiar las diferencias pre y post intervención psicológica online durante 9 meses en un joven jugador de fútbol profesional. Se parte de la demanda del deportista para mejorar su fortaleza mental y conseguir la titularidad en el equipo, ya que después de 2 meses de temporada, era suplente y se sentía "débil psicológicamente" para afrontarlo. Para ello, se evaluó al jugador con el CPRD, autorregistros y observaciones de partidos. La intervención se realizó por medio de videoconferencias, mensajería instantánea y correo electrónico. Principalmente, se aplicó técnicas cognitivo-conductuales, como la relajación, las autoinstrucciones, el planteamiento de creencias potenciadoras y establecimiento de objetivos. Los resultados muestran una mejora de las habilidades psicológicas evaluadas del futbolista, así como el logro de la titularidad en el equipo. Se concluye que la intervención psicológica online es eficaz para la mejora de las habilidades psicológicas y el rendimiento deportivo a lo largo de la temporada, en el que el psicólogo del deporte tiene que aprender a darle uso e integrarlas dentro de su desempeño profesional.

PALABRAS CLAVES: intervención psicológica, online, fútbol, nuevas tecnologías.

\section{Remote psychological intervention with a professional footballer: case study}

ABSTRACT: The purpose of this paper was to assess the changes from baseline in a young professional football player after an online psychological intervention for 9 months. The athlete demanded the intervention to improve his mental strength and be able to be in the starting eleven of the team, because after 2 months of season he was a substitute and felt "psychologically weak" to face it. The player was evaluated using the CPRD questionnaire, self-reports, and viewing matches. The intervention was conducted through video conferences, instant messages, and emails. Cognitive behavioural techniques such as relaxation, self-instruction, empowerment approach, and goal setting were mainly used. The results showed an improvement in the assessed psychological skills of the football player, who also played in the starting lineup. It is concluded that online psychological intervention is effective to improve psychological skills and sports performance throughout the season, and sport psychologists have to learn how to make use of it and integrate it into their professional practice.

KEYWORDS: psychological intervention, online, football, new technologies.

\section{Intervenção psicológica remota com um futebolista profissional: estudo de caso}

RESUMO: O objetivo do presente trabalho é estudar as diferenças entre a pré-intervenção e a pós-intervenção psicológica on-line durante nove meses num jovem jogador de futebol profissional. Tem por base a intenção do atleta de melhorar a sua força mental e conseguir ser titular da equipa, uma vez que, após dois meses de temporada, se mantinha como suplente e sentia-se "psicologicamente fraco" para enfrentar a situação. Para isso, o jogador foi avaliado através das CPDD, de autorregistos e observação de jogos. A intervenção foi realizada por videoconferência, mensagens instantâneas e e-mail. Foramaplicadas principalmentetécnicas cognitivas-comportamentais, como relaxamento, autoinstrução, abordagem de crenças potenciadoras e estabelecimento de objetivos. Os resultados mostram uma

\footnotetext{
David Peris Delcampo es psicólogo acreditado como experto en psicología del deporte (COP). Profesor de la Universitat de València · Associació de Psicologia de l'Esport de la Comunitat Valenciana. Vicepresidente de la Federación Española de Psicología del Deporte. También es Entrenador Nacional de Fútbol y de Fútbol Sala.
}

La correspondencia sobre este artículo debe enviarse a David Peris Delcampo a Avda. Jaume I, nº 26, 10 H, 12530 Burriana (Castellón). E-mail:

campo@hotmail.com 
melhoria das competências psicológicas avaliadas do jogador de futebol, bem como a conquista da titularidade na equipa. Conclui-se que a intervenção psicológica on-line é eficaz para a melhoria das competências psicológicas e do desempenho desportivo ao longo da temporada, na medida em que o psicólogo desportivo aprenda a usá-la e integrá-la no desempenho profissional.

PALAVRAS-CHAVE: intervenção psicológica, on-line, futebol, novas tecnologias.

Artículo recibido: 11/07/2019 | Artículo aceptado: 02/11/2019

Las posibilidades de intervención psicológica actuales precisan de profesionales debidamente preparados y acreditados que se adapten a las demandas reales del cada vez más cambiante mundo del deporte (Cantón, 2016). En este sentido, el uso adecuado de las nuevas tecnologías en Psicología supone un avance en las posibilidades de intervención, aunque es necesario hacer un buen uso de estas nuevas herramientas (Guillén, Grau-Alberola y Cortell, 2018), respetando la legalidad, actuando de manera profesional, respetando el código deontológico y demás criterios profesionales en beneficio del cliente (Consejo General de la Psicología de España, 2017).

En el fútbol, en los últimos años se ha asentado el rol y funciones del psicólogo del deporte, especialmente en el fútbol formativo y en menor medida en el fútbol profesional (Garcia-Naveira, 2018), aunque no es habitual la publicación de trabajos que utilizan las nuevas tecnologías como medio de evaluación e intervención de los deportistas, a pesar que se cuentan con los medios adecuados para ello.

La posibilidad de realizar videoconferencia o el empleo del teléfono móvil, mensajería instantánea o el correo electrónico, facilita la optimización de la intervención psicológica "a distancia" en el deporte, en ocasiones debido a la distancia geográfica o la limitación de horarios entre el profesional y el cliente, con un trato muy personalizado, aún sin poder realizar sesiones presenciales (Vives, 2014).

Además, en España y otros países, las opciones de recoger información a distancia son cada vez mayores al disponer de plataformas digitales donde se pueden ver todos los partidos de liga de fútbol (e.g., MoviStar+, DAZN, BEIN y GolTV), otras aplicaciones relacionadas con el Big Data (e.g., Media Coach, WyScout, Transfermatkt, Whoscored y SofaScore, Optapro) o para analizar videos (e.g., NacSport, LongoMatch y Eric Sport), lo que aumenta considerablemente la recogida de información del jugador y/o equipo de fútbol (Memmbert y Raabe, 2018).

Partiendo de este panorama, el presente trabajo expone un estudio con un joven jugador de fútbol profesional, realizando una evaluación pre y pos intervención psicológica online, cuestión que requiere de más trabajos aplicados al respecto. Además, el estudio de caso único es una metodología que empieza a ser muy valorada, ya que proporciona un marco referencial para los profesionales del área, reduciendo la distancia entre la investigación y la práctica (Olmedilla y Dominguez-Igual, 2016). Por ello, se plantea la hipótesis de que la intervención psicológica online y personalizada, mejorará las variables psicológicas estudiadas y contribuirá al logro de los objetivos del deportista.

\section{Método}

Se utiliza un diseño de caso único $A-B$, con fase de seguimiento cualitativa posterior (León y Montero, 2015) de triangulación metodológica (Castañer, Camerino y Anguera, 2013) utilizando métodos cuantitativos y cualitativos. El tratamiento de datos se realizó a través de análisis visual por medio del test-retest cuantitativo (Bono y Arnau, 2014).

\section{Participantes}

El participante fue un futbolista profesional de 21 años (internacional en las categorías inferiores de la Selección Española) de un equipo que la temporada anterior estaba en Segunda División. Ficha por su actual equipo después de ser un jugador relevante en su anterior club con las expectativas de ser importante y continuar su proyección deportiva. Anteriormente había jugado en las categorías inferiores de uno de los clubs más importantes de España y había sido cedido en dos ocasiones para, en esta temporada, quedar libre y firmar por su actual equipo. El jugador compagina su actividad deportiva con sus estudios universitarios.

A modo de referencia, el jugador entrena 4 días a la semana por las mañanas, en sesiones de 2 horas, más el partido del campeonato. Además, asiste a clase de lunes a viernes por la tarde, con una dedicación de cuatro horas diarias y su correspondiente preparación de los exámenes.

La intervención psicológica fue realizada por un psicólogo acreditado como experto en psicología del deporte por el 
Consejo General de la Psicología del España, que además tiene la titulación de Técnico Deportivo Superior en Fútbol (Entrenador Nacional, UEFA Pro), con más de 20 años de experiencia profesional en el fútbol y otros deportes.

\section{Instrumentos}

La evaluación cuantitativa se realizó con el Cuestionario de Características Psicológicas para el Rendimiento Deportivo (CPRD) de Gimeno-Marco y Buceta (2010), de 55 ítems de cinco alternativas, que cuenta con los siguientes factores: Influencia en la Evaluación del Rendimiento (impacto de las situaciones competitivas y su evaluación por parte del deportista y otras personas cercanas), Control del Estrés (manejo de situaciones estresantes), Motivación (importancia sobre la actividad deportiva en general y en su día a día), Habilidad Mental (habilidades mentales que dispone el deportista) y Cohesión de Equipo (disposición a trabajar en equipo). El instrumento en su totalidad tiene un coeficiente a de Cronbach de .85. Para la interpretación de los datos se utilizaron los baremos que presentan los autores para deportistas de élite.

Las videoconferencias se realizaron por medio de un ordenador personal Macintosh y/o tablets Ipad Mini utilizando el programa "Skype", mientras que para la mensajería instantánea se utilizó la aplicación Whatsapp y el servidor de correo electrónico habitual de cada uno.

\section{Procedimiento}

El futbolista contactó con el psicólogo del deporte al considerar que en pretemporada y los dos primeros partidos de liga no era "el jugador importante que debería ser", "quería ser mejor" y "un 'toro' mentalmente". No se veía titular indiscutible (jugó desde el banquillo los dos partidos oficiales) en su equipo, el cual sacó cuatro puntos en dos jornadas de liga. Quería funcionar mejor a nivel mental, porque se sentía débil al no ser titular en el equipo. A partir de aquí, por teléfono, se concretan las condiciones de trabajo que, debido a la distancia física entre ambos (diferentes autonomías), se realizaría a distancia (online), hablando por videoconferencia, una vez a la semana en el primer mes, y después, dos veces al mes y con contacto directo vía mensajería instantánea, además de utilizar el correo electrónico para poder realizar "tareas para casa". También, el psicólogo del deporte vería los partidos en los que jugaría el futbolista que fueran retransmitidos por televisión (en directo y si no en diferido), a fin de analizar su comportamiento futbolístico relacionado con las variables psicológicas trabajadas.

Previamente se le explicó las características de la intervención firmando el consentimiento informado en base a lo recomendado por el Comité de Ética de la Universidad de Valencia.

En total, se realizaron, entre septiembre y junio, 22 sesiones por videoconferencia (desarrolladas entre semana, entre las cinco y las ocho de la tarde, según agenda de ambos) de aproximadamente 50 minutos de duración cada una; alrededor de 60 interacciones vía Whatsapp; y un total de 43 conversaciones mediante correo electrónico. A principios de julio se realizó otra sesión de videoconferencia de cierre de la temporada. En la Tabla 1 se presenta un resumen del contenido y temáticas trabajadas en las diferentes sesiones.

Aunque el psicólogo del deporte tenía conocimiento del fútbol (y eso ayudó a comentar situaciones deportivas y manejo de conceptos), el trabajo se centró en aspectos psicológicos relacionados con la demanda del jugador, y que era el que proporcionaba, si se requería, los criterios técnicos/ tácticos y otros más específicos del fútbol, aun entendiendo que no existen "partes por separado", sino que todo está interrelacionado, por lo que se analizaba conjuntamente respetando el criterio deportivo del futbolista y manejando las variables psicológicas en base a eso.

La intervención se realizó mediante el enfoque coaching (Peris-Delcampo, 2016) por medio del cual el protagonista es el futbolista y el experto en coaching facilita las condiciones de manera no directiva para que el deportista encuentre sus soluciones en base a unos objetivos propuestos. También, se tuvo en cuenta ciertas variables psicológicas implicadas en el rendimiento, las ideas potenciadoras, los objetivos planteados, las estrategias fisiológicas de control de la activación (control de la respiración + autoinstrucciones) y las relacionadas con el autorrefuerzo y refuerzo a través del registro realizado para cada partido para después de las competiciones que englobaba lo anterior. Todo ello se detalla a continuación.

El punto de partida de esta intervención psicológica tiene que ver con el manejo adecuado de variables psicológicas relacionadas con el rendimiento deportivo (Buceta, 2016; Peris-Delcampo, 2008). Es decir, cuando se trabajaban aspectos relacionados con el juego, se "pensaba" en variables psicológicas que en este caso eran: (1) autoconfianza o la percepción realista de que haciendo lo que uno es capaz de hacer puede lograr los objetivos que se propone, (2) nivel de activación o el grado de excitación (fisiológica y/o cognitiva) a la hora de enfrentarse a una situación, (3) atención/concentración o la selección de estímulos adecuados, (4) control del estrés, entendido como 
Tabla 1. Sesiones de trabajo y objetivos de la intervención psicológica a distancia $(n=1)$

\begin{tabular}{|c|c|c|}
\hline Fechas & Sesiones realizadas & Objetivos \\
\hline 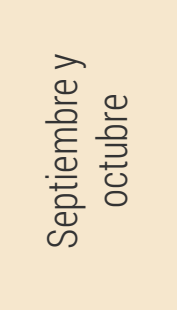 & $\begin{array}{l}6 \text { sesiones videoconferencia. } \\
15 \text { correos electrónicos (de ida y } \\
\text { vuelta) } \\
21 \text { interacciones por Whatsapp. } \\
\text { Visionado de } 10 \text { partidos de liga y } 1 \\
\text { de Copa del Rey. }\end{array}$ & $\begin{array}{l}\text { Definir la demanda. } \\
\text { Fase pre test. } \\
\text { Fase educativa (ideas potenciadoras, establecimiento } \\
\text { de objetivos) } \\
\text { Creación del autorregistro. } \\
\text { Análisis del trabajo realizado }\end{array}$ \\
\hline 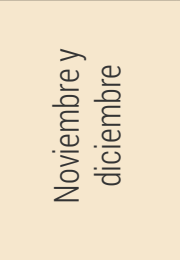 & $\begin{array}{l}4 \text { sesiones videoconferencia } \\
8 \text { correos electrónicos } \\
8 \text { interacciones por whatsapp } \\
\text { Visionado de } 8 \text { partidos de liga }\end{array}$ & $\begin{array}{l}\text { Desarrollo de ideas potenciadoras. } \\
\text { Implementación de las técnicas de respiración y de } \\
\text { autoinstrucciones. } \\
\text { Autorregistro. } \\
\text { Seguimiento de los objetivos. }\end{array}$ \\
\hline 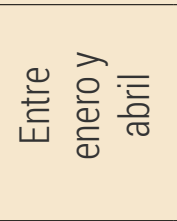 & $\begin{array}{l}6 \text { videoconferencias } \\
12 \text { int. correo electrónico } \\
16 \text { int. por whatsapp } \\
\text { Visionado de } 16 \text { partidos de liga }\end{array}$ & $\begin{array}{l}\text { Trabajo de ideas potenciadoras. } \\
\text { Aplicación respiración y autoinstrucciones. } \\
\text { Autorregistro. } \\
\text { - Revisión y consolidación objetivos. }\end{array}$ \\
\hline 站 亭·읕 & $\begin{array}{l}4 \text { videoconferencias } \\
8 \text { int. correo electrónico } \\
14 \text { int. whatsapp } \\
\text { Vis. de } 6 \text { partidos de liga }\end{array}$ & $\begin{array}{l}\text {-Seguimiento del plan de trabajo. } \\
\text {-Manejo ideas potenciadoras y objetivos para controlar } \\
\text { situaciones estresantes, centrándose en "su trabajo". } \\
\text { - Envío y cumplimentación del pos test. }\end{array}$ \\
\hline 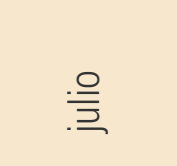 & $\begin{array}{l}1 \text { entrevista por videoconferencia } \\
4 \text { interacciones por whatsapp }\end{array}$ & $\begin{array}{l}\text {-Valoración, refuerzo y consolidación del trabajo reali- } \\
\text { zado. } \\
\text { - Análisis del pos test. }\end{array}$ \\
\hline
\end{tabular}

la movilización de recursos fisiológicos y psicológicos ante una situación que se percibe como amenazante, (5) motivación o la fuerza/interés por conseguir algo de manera adecuada, (6) comunicación o la forma de interacción de los mensajes propios y con los otros, y (7) cohesión de equipo, entendido como la capacidad de funcionar como grupo juntos en busca de objetivos comunes.

La técnica de establecimiento de objetivos (Locke y Latham, 1990; Morelló, Bert y Navarro, 2018; Peris-Delcampo, 2006), se usó pidiendo al jugador que planteara los objetivos de la temporada (que inicialmente eran de resultado) y trabajando conductas concretas (objetivos de realización o de tarea) que se plasmaron en un autorregistro de partido, con tres objetivos en defensa (por ejemplo "presión tras pérdida") y tres en ataque (por ejemplo, "elegir entre pasar o driblar") que el futbolista enviaba por correo y era comentado por videoconferencia en las sesiones o por "whatsapp" (recibiendo de esta manera feedback del partido).

El planteamiento de creencias potenciadoras (Ellis, 1980; Peris-Delcampo, 2019), se realizaba en relación con el establecimiento de objetivos, de manera que, el tener claro qué podía hacer en cada momento y cómo evaluar- los, le daba seguridad y le hacía centrarse en su "trabajo" (estar pendiente de lo que le ayudaba a rendir bien). En principio, mediante preguntas de reflexión, se le cuestionó qué es lo que le hacía funcionar mejor. Tras dos sesiones (en la primera fase de la temporada), después de que el futbolista llegara a la conclusión de que podía centrarse en lo que realmente dependía de él, se le plantearon las diez ideas potenciadoras (Tabla 2), utilizándolas cuando se consideró necesario, para reenfocar el plan de acción. Además, para la mejora los conceptos de juego que él había decidido relacionados con los objetivos (por ejemplo, "presión tras pérdida al poseedor del balón"), se le instó a que se planteara mensajes a utilizar durante el partido que fueran potenciadores, convirtiéndolo en autoinstrucciones (Weinberg y Gould, 1995) para momentos clave del partido.

La estrategia conjunta de control de la respiración y de cogniciones (según las recomendaciones de Labrador, Cruzado y Muñoz, 2001), se realizó por medio de una primera fase educativa, en la que se le explicó por videoconferencia al jugador cómo respirar con el diafragma, para después utilizarla durante muchas veces al día, siempre que pudiera. Posteriormente, la aplicaba en los entrena- 
Tabla 2. Ideas potenciadoras utilizadas en la intervención (Peris-Delcampo, 2019)

\begin{tabular}{ll}
\hline \multicolumn{1}{c}{ Idea Potenciadora } & \multicolumn{1}{c}{ Significado } \\
\hline 1. Esfuerzo Realista. & $\begin{array}{l}\text { Pienso en lo que yo puedo hacer y me esfuerzo al máximo que yo deseo, aceptando que las circunstancias pueden ser } \\
\text { más o menos favorables a mis actos. }\end{array}$ \\
\hline 2. Disfrute. & Deseo lograr mi objetivo. Disfruto yendo hacia el objetivo que yo decido. \\
\hline 3. Propia elección. & Los demás no saben realmente hasta dónde puedo llegar. Yo elijo mi esfuerzo y mi camino y decido dónde está mi límite. \\
\hline 4. Disputa sana. & En este mundo hay sitio para todos. Pelearé hasta donde yo decida, aceptando las reglas del juego con deportividad. \\
\hline 5. Aprender del camino. & $\begin{array}{l}\text { Un fracaso es una oportunidad de aprender. Superar fracasos me hace cada vez más fuerte. El fracaso forma parte del } \\
\text { camino necesario para alcanzar mi éxito. }\end{array}$ \\
\hline 6. Auto éxito. & Mis triunfos son fruto de mi propia elección. El éxito o el fracaso es mío y yo decido cuál es el camino para ello. \\
\hline 7. iPresente! & El presente es el único lugar donde actuar. \\
\hline 8. Colaboración. & Estar en contacto con los demás nos ayuda a crecer. Es beneficioso para todos colaborar con otros de forma constructi- \\
\hline va. Puedo elegir cómo y hasta dónde colaborar con otros.
\end{tabular}

mientos asociada a una autoinstrucción relacionada con el juego, y después ya en momentos puntuales de la competición si la consideraba oportuna. Ciertamente, la utilizó pocas veces, aunque decía que, el conocer esta estrategia, le daba seguridad para afrontar cada partido.

En la primera sesión se realizó la evaluación, en que la corrección del cuestionario empleado, se cumplimentó en casa posteriormente y fue enviado y recibido por correo electrónico. Posteriormente, se establecieron los objetivos de intervención (la demanda principal era "sentirse fuerte mentalmente para acabar la temporada como titular") y las estrategias psicológicas utilizadas donde, tras las conversaciones con el deportista y ver y comentar dos partidos se enfatizó inicialmente la mejora del "nivel de activación" y la "orientación hacia la tarea sobre todo los momentos psicológicamente más intensos" (en base a lo visto en los dos partidos y las conversaciones con el deportista). Se esperaba que, funcionando a un buen nivel psicológico, a partir de sus carencias (valoradas a través del pre test y las conversaciones y estrategias elegidas durante la intervención), manejaría adecuadamente las variables psicológicas e ideas potenciadoras y tendría más posibilidades de sentirse titular.

En la siguiente fase de la temporada, se siguió con el plan de trabajo propuesto, analizando el autorregistro para cada partido, la implantación y debate de ideas potenciadoras a través del enfoque coaching (Peris-Delcampo, 2016) y se incorporó una estrategia de respiración para el control de su nivel de activación junto con autoinstrucciones pactadas para el partido en juego.
Después del "mini parón" de Navidad y hasta la finalización de abril, se siguió con el plan previsto. En cada sesión, trabajando las ideas potenciadoras, comentando específicamente situaciones relacionadas con el nivel de activación valorando el grado de utilización de la técnica de respiración y de las autoinstrucciones, relacionadas con los objetivos que se planteaba en el registro.

En la última parte de la temporada, aunque se siguió el mismo esquema previsto anteriormente, se enfatizó mucho más en manejar bien los aspectos psicológicos relacionados con el juego y pensamientos relacionados con cumplir las expectativas de final de temporada, donde pensaba que un buen rendimiento le abriría las puertas a otro equipo, reforzando las tareas hechas hasta ese momento y que "hiciera su trabajo", es decir, lo que dependía de él, buscando un nivel óptimo de funcionamiento.

En la primera semana de julio, después de casi tres semanas de finalización de la temporada, se realizó una sesión de seguimiento para reforzar el trabajo realizado (buscando su consolidación).

Desde el principio de la intervención, el futbolista mandaba su valoración de cada partido de manera breve por Whatsapp y de forma bastante más detallada por correo electrónico, dándole un feedback posterior al futbolista.

Como ejemplo, una sesión tipo de las realizadas por videoconferencia (aproximadamente de 50 minutos de duración) constaba de las siguientes fases: (1) comentarios generales sobre cómo se encontraba y cómo iba funcionando (valorando las variables psicológicas y las ideas 
potenciadoras), (2) análisis del autorregistro, (3) desarrollo de ideas potenciadoras y (4) plan de trabajo para el futuro. Cada sesión, aunque se seguía la mayoría de las veces esta estructura, era diferente a las otras ya que se adaptaba a lo que iba ocurriendo en cada momento.

\section{Resultados}

Los resultados cuantitativos del CPRD (Figura 1) muestran una mejora significativa de los factores Influencia en la Evaluación del Rendimiento, Control del Estrés y Habilidad Mental, donde se pasó del percentil 50 a 70, de 30 a 80, y de 65 a 90, respectivamente; una disminución en la Motivación (que estaba muy alta, pasando de 90 a 75) y un mantenimiento en un alto percentil de Cohesión de Equipo (en ambos casos percentil 70).

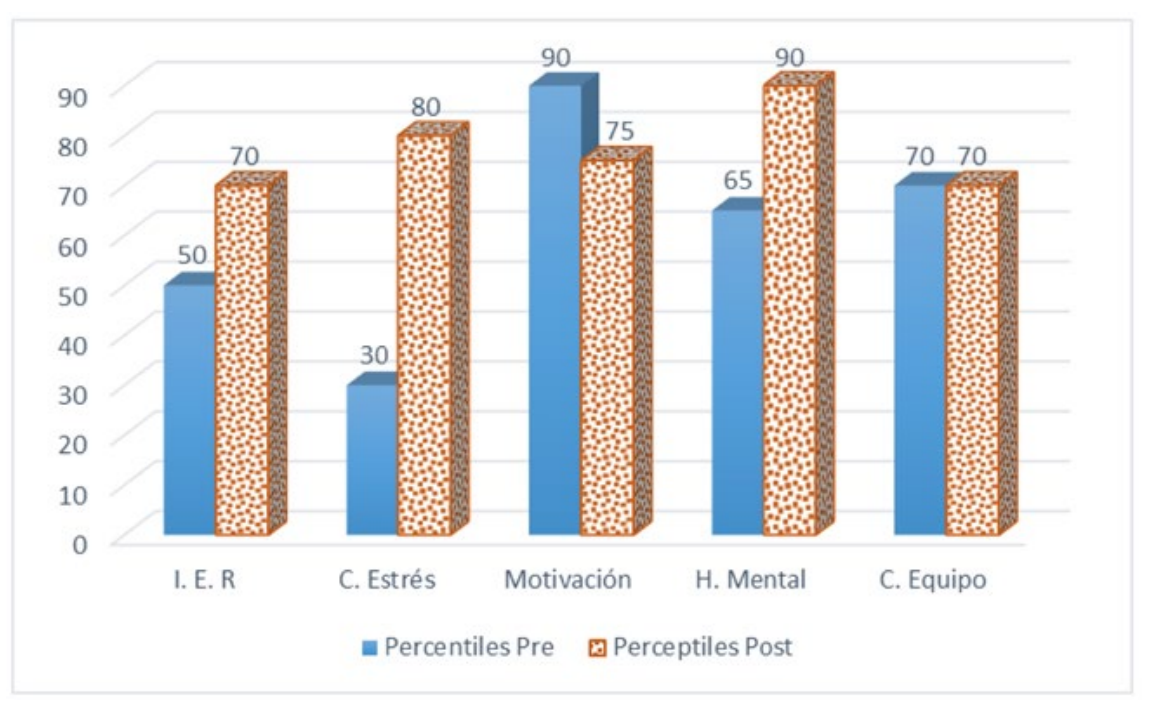

Figura 1.- Puntuaciones centiles pre y post para los cinco factores del CPRD. Influencia en la Evaluación del Rendimiento (I.E.R.), Control del Estrés (C. Estrés), Motivación, Habilidad Mental (H. Mental) y Cohesión de Equipo (C. Equipo).

Por su parte, en relación a las impresiones del futbolista, manifestaba que "se sentía con estrategias para afrontar los momentos más complicados de los partidos", de manera que estaba "más seguro en los momentos más difíciles" porque "sabía qué podía hacer para encontrar su estado óptimo de funcionamiento", aceptando "mejor las circunstancias buscando soluciones ante las posibles dificultades" (en lugar de centrarse en los problemas).

Además, y en relación al autorregistro donde después de cada partido valoraba la consecución de tres objetivos en ataque y tres en defensa, manifestó que "le había servido para controlar mejor lo que tenía que hacer en cada partido", lo que hacía que se "sintiera y funcionara mejor durante los partidos".

La consecuencia de todo ello, a nivel deportivo, fue que acabó el año siendo titular, según su percepción, jugando los últimos 29 partidos de la temporada desde el principio en los partidos que disputó con su equipo.

\section{Discusión}

Los resultados muestran una mejora de las habilidades psicológicas del jugador durante el juego, cumpliéndose así con el objetivo inicial. Esto se concluye tanto por las puntuaciones del cuestionario (sobre todo en el aumento de "habilidad mental" y "control del estrés") como por lo que decía el futbolista, manifestando sentirse mejor y más competente jugando a su óptimo nivel.

El aumento en la puntuación "Habilidad Mental", está en consonancia con la mejora manifiesta del futbolista sobre todo en las situaciones más complicadas, así como su autorregulación en relación con las variables psicológicas relacionadas con el juego. De esta forma, al aprender a establecer mejor los objetivos (sobre todo convirtiendo los resultados en tareas), adquirir habilidades de control de la activación, plantearse autoinstrucciones relacionadas con el juego y el manejo adecuado de ideas potenciadoras, funciona mejor durante el juego por lo que también tiene una mayor percepción de dominar habilidades psicológicas que le son útiles.

Asimismo, también la diferencia al alza de las puntuaciones de "Control del Estrés" e "Influencia en la Evaluación del Rendimiento", están relacionadas con tener claro qué es lo que tiene que hacer en cada momento, asumiendo las decisiones (percepción de control), o el saber autorregularse su nivel de activación, sobre todo en momentos complicados o tras cometer un error o una acción que ha salido mal.

Por su parte, una disminución de la motivación está en relación al adecuado planteamiento de objetivos siendo así más realista, centrándose en lo que depende de él. De esta manera, parece indicar que en el pre test, el futbolista tenía una motivación de baja calidad enfocándose a objetivos mal planteados.

Estos resultados del CPRD están en relación a las conclusiones sacadas de las entrevistas con el futbolista y el análisis visual de los partidos ya que, por ejemplo, decía que se "regulaba mejor en el partido" (refiriéndose a la variable "nivel de activación"), sabía "qué podía hacer y no hacer en cada momento" ("autoconfianza", "atención" hacia estímulos relevantes, "enfoque motivacional orientado a la tarea"), o podía "funcionar bien en momentos complicados" ("control del estrés").

Además, y en relación a las ideas potenciadoras, también mostró una mejora, en coordinación con las demás estrategias psicológicas planteadas, de manera que era capaz de centrarse en lo que realmente dependía de él ("iPresente!", 
"Auto éxito"), hacía en cada momento lo que creía que era mejor ("Propia elección", "Esfuerzo realista") o analizaba lo que había ocurrido buscando soluciones de manera constructiva ("Aprender del camino", "Aceptación").

El profesional de la Psicología del Deporte debe estar cada vez más preparado para intervenir con eficacia adaptándose a las características y demanda de la intervención, adquiriendo y utilizando las competencias profesionales adecuadas a su trabajo (Peris-Delcampo y Cantón, 2018). Actualmente, es posible hacer eficaces intervenciones a distancia utilizando los medios tecnológicos disponibles. Para ello, el psicólogo del deporte tiene que aprender a dar uso a las nuevas tecnologías y respetar la legislación presente de su uso.

La intervención a distancia, por su parte, presenta dificultades en relación a la intervención presencial ya que ésta tiene un trato más directo con el deportista, realizando entrevistas más cercanas (lo que ayuda también a captar más detalles). También la intervención online presenta ventajas como la adecuación a horarios más flexibles, la posibilidad de reducir el coste de las sesiones por medio de, por ejemplo una psicología low cost (Vives, 2014). No obstante, a eso, actualmente y con los medios técnicos (posibilidad de videoconferencia, ver los partidos on line, correo electrónico, mensajería...) facilita mucho que la intervención se centre en información relevante y en estrategias individualizadas.

Respecto a la actual intervención y como propuesta a futuros trabajos similares, hubiera sido más adecuado llevar un registro por escrito de las evaluaciones post partido (en relación a los tres objetivos ofensivos y tres defensivos), quizás por medio de una gráfica, un análisis cualitativo de las ideas potenciadoras pre y post (explicando pensamientos antes y después de la intervención para cada una de las creencias) y quizás también una manera más objetiva de medir el comportamiento psicológico (por medio de variables) en la competición (a través de una autoevaluación del 1 al 10 para cada variable o bien otro indicador que se hubiera pactado).

Estudios de este tipo, aunque tienen las limitaciones propias de los estudios de caso (León y Montero, 2015), como son el sesgo del profesional que interviene, la dificultad de controlar todas las variables intervinientes o la dificultad de generalizar las conclusiones al tratarse del análisis de un solo sujeto, son necesarios para difundir la parte práctica a los psicólogos del deporte ofreciendo herramientas útiles a los profesionales aplicados en este campo.

Es necesario tener en cuenta, además, que en intervenciones psicológicas a distancia, se precisa ajustarse al código deontológico del profesional de la psicología (Consejo General de la Psicología de España, 2017), explicar previa- mente las condiciones del trabajo y aprovechar los medios de los que se dispone buscando facilitar la relación y el funcionamiento del deportista, a través de una buena práctica profesional desarrollando las competencias adecuadas (Peris-Delcampo y Cantón, 2018).

\section{Referencias}

Bono, R. y Arnau, J. (2014). Diseños de caso único en ciencias sociales y de la salud. Madrid, España: Editorial Síntesis.

Buceta, J. M. (2016). Variables psicológicas relacionadas con el rendimiento deportivo.Madrid, España: Universidad Nacional de Educación a Distancia. http://e-spacio.uned.es/fez/view. php?pid=tesisuned:Psicologia-Phgarcia

Cantón, E. (2016). La especialidad profesional en Psicología del Deporte. Revista de Psicología aplicada al Deporte y al Ejercicio Físico, 1, E2. https://doi.org/10.5093/rpadef2016a2

Castañer, M., Camerino, O. y Anguera, M. T. (2013). Métodos mixtos en la investigación de las ciencias de la actividad física y el deporte. Apunts. Educación Física y Deportes, 112(2), 31-55. https://doi.org/10.5672/apunts.2014-0983.es.(2013/2).112.01

Consejo General de la Psicología de España (2017). Guía para la práctica de la telepsicología. Madrid, España: Autor. https://doi. org/10.23923/cop.telepsicologia.2017

Ellis, A. (1980). Razón y Emoción en Psicoterapia. Bilbao, España: Desclée de Brouwer.

García-Naveira, A. (2018). Pasado, presente y futuro del psicólogo del deporte en el fútbol español. Revista de Psicología aplicada al Deporte y al Ejercicio Físico, 3, e2. https://doi.org/10.5093/rpadef2018a8

Gimeno-Marco, F. y Buceta, J. M. (Eds.). (2010). Evaluación psicológica en el deporte: cuestionario de «características psicológicas relacionadas con el rendimiento deportivo» (CPRD). Madrid, España: Dykinson.

Guillén, V., Grau-Alberola, E. y Cortell, M. (2018). Reflexiones acerca de la utilización de las Tecnologías de la Información y la Comunitación (TICs) en Psicología Clínica: eficacia, ventajas, peligros y líneas futuras de investigación. Informació Psicològica, 116, 121-132

Labrador, J., Cruzado, J. A. y Muñoz, M. (coord.) (2001). Manual de Técnicas de modificación y terapia de conducta. Madrid, España: Pirámide.

León, O. G. y Montero, I. (2015). Métodos de investigación en Psicología y Educación: las tradiciones cuantitativa y cualitativa, (4 ${ }^{\circ}$ Ed.). Madrid, España: Mc Grawhill.

Locke E. A. y Latham G. P. (1990). A Theory of Goal Setting and Task Performance. New York: Prentice-Hall, Englewood Cliffs.

Membert, D. y Raabe, D. (2018). Data analytics in football: positional data collection, modeling and analysis. Nueva York, NY: Routledge.

Morelló, E., Bert, B. y Navarro, S. (2018). Establecimiento de objetivos en el curriculum formativo de los futbolistas. Revista de Psicología aplicada al Deporte y al Ejercicio Físico, 3, e3. https:/l doi.org/10.5093/rpadef2018a7

Olmedilla, A. y Dominguez-Igual, J. (2016). Entrenamiento psicológico para la mejora de la atención y la autoconfianza en un 
futbolista. Revista de Psicología Aplicada al Deporte y al Ejercicio Físico, 1, e4. https://doi.org/10.5093/rpadef2016a4

Peris-Delcampo, D. (2006). El establecimiento de objetivos como estrategia psicológica para el entrenador de fútbol. El entrenador español de Fútbol, 109. https://doi.org/10.3900/fpj.7.2.112.s

Peris-Delcampo, D. (2008). El entrenamiento psicológico en fútbol: variables implicadas. Tui, España: MC Sports.

Peris-Delcampo, D. (2016). Nociones básicas de Psicología del Deporte y Coaching para Gestores de Empresas Deportivas. Almería, España: Círculo Rojo. https://doi.org/10.7203/anuari.psicologia.19.1.307

Peris-Delcampo, D. (2019). Ideas potenciadoras vs ideas limitantes. Máster en Coaching y Liderazgo de la Universidad de Valencia. Valencia, España: Fundación Universidad Empresa Adeit.
Peris-Delcampo, D. y Cantón, E. (2018). El perfil profesional del psicólogo especialista en psicología del deporte en fútbol. Revista de Psicología Aplicada al Deporte y el Ejercicio Físico, 3, e9. https://doi.org/70.5093/rpadef2018a6

Vives, J. (2014). Psicología low cost: ejemplos de intervenciones eficientes en deportes de equipo. En J. Cruz y J. Riera, International Conference of Psychology Applied to Soccer \& Team Sports (CIPAF) y XXIV Jornades ACPE. Associació Catalana de Psicologia de l'Esport. Barcelona, del 5 al 7 de noviembre de 2014. https://doi.org/10.32800/amz.2017.15.0001.

Weinberg, R. S. y Gould, D. (1995). Fundamentos de Psicología del Deporte. Barcelona, España: Ariel. 